\title{
A Model of Public Announcement System's Dynamics Control in Smart City Environments
}

\author{
Ante Grgat ${ }^{1,2}$, Ivan Djurek ${ }^{2,}$, Mia Suhanek ${ }^{2}$ \\ ${ }^{1}$ Ericsson Nikola Tesla, Zagreb, Croatia \\ ${ }^{2}$ Faculty of Electrical Engineering and Computing, University of Zagreb, Zagreb, Croatia
}

Email address:

ivan.djurek@fer.hr (I. Djurek)

${ }^{*}$ Corresponding author

\section{To cite this article:}

Ante Grgat, Ivan Djurek, Mia Suhanek. A Model of Public Announcement System's Dynamics Control in Smart City Environments. American Journal of Environmental Science and Engineering. Special Issue: Smart Cities - Innovative Approaches.

Vol. 3, No. 4, 2019, pp. 112-116. doi: 10.11648/j.ajese.20190304.17

Received: November 13, 2019; Accepted: December 2, 2019; Published: December 17, 2019

\begin{abstract}
Nowadays, each individual is exposed to noise on a daily basis. Long exposure to noise pollution can be manifested through several health concerns such as bad mood, fatigue, insomnia, headache and loss of concentration, which can then cause reduced work ability and finally permanent hearing impairment. When considering urban areas, the most common type of noise source is traffic. Public announcement system are a vital and much needed part of every urban area and thus it should be constructed in a way that it delivers relevant information in a clear and understandable way while not disturbing the residents. Therefore, this paper proposes a model of public announcement system in urban places which aims to reduce unexpected and sudden loudness changes. The system is envisaged for public places, such as open bus stations surrounded with residential and commercial buildings. Several studies have shown that the residents of these buildings find sudden announcements very annoying and disturbing. The goal of this research is to reduce the sound level while retaining the principal loudness level. The study has shown that with the appropriate signal processing which includes a compressor and a limiter, these types of announcements can be made less annoying and disturbing for urban residents.
\end{abstract}

Keywords: Public Announcement System, Loudness Changes, Signal Processing, Smart Cities

\section{Introduction}

Noise pollution is a "hot" topic today and each individual is exposed to noise on a daily basis. On the other hand, noise pollution is often overlooked and neglected when compared to other different environmental (e.g. air, water or soil pollution). Long exposure to noise pollution can be displayed as a bad mood, fatigue, insomnia, headache and loss of concentration, which can cause reduced work ability and ultimately permanent hearing impairment [1-3]. In urban areas there are different types of noise however the "dominant" noise source is traffic [4-6]. Bearing in mind all of the aforementioned issues in urban areas regarding noise, public announcement system should be designed in a way that the majority of population does not perceive it as noise and therefore ignore it. Public announcement systems represent a significant part of every urban area and they should deliver relevant information in a clear and nondisturbing way for the residents.

Thus, this paper proposes a model for public announcement (PA) system's noise control in urban areas based on distributed microphones. Signals from these microphones are used as sensing signals for loudness control of public announcement systems, for example at open or semi-open bus stations. Residents who live close to these types of buildings are often exposed to traffic noise and in addition, from noise coming through public announcement systems incorporated in these buildings. Public announcement systems, used for announcing departure and arrival of buses, include spoken announcements after an initial signal tone. These announcements are not constant and, from the residents' point of view, appear randomly, and therefore are often perceived as unexpected and sudden loudness changes.

Figure 1 shows a neighborhood in Zagreb close to the central bus station [7]. The distance between the platforms and 
closest commercial and residential buildings is around 150 meters. The bus station uses a loud PA system for announcements of arrivals and departures of buses. In order to achieve enough loudness in terms of speech intelligibility of the announcer's voice around the noisy environment of the bus station, its voice needs to be over amplified. The residents in nearby buildings perceive this as unexpected and sudden loudness changes which can be very annoying. A short survey among residents whose apartments are in direct line of sight to the station, showed that residents are used to surrounding traffic and other noises, however they find sudden changes of loudness very disturbing. Measurements showed that the announcer's voice level above the surrounding noise is 20 to $30 \mathrm{~dB}$. Our previous research showed that these salient sounds tend to be very annoying and disturbing, especially if level differences among peak level and average level are high [8]. The people tend to get used to surrounding noise, if it is not too high, however sudden loudness changes are often perceived as annoying, especially at evening, night and morning, when surrounding noise levels are low [9]

Therefore, the goal is to adjust the level of the PA system in the bus station in a way that there are no sudden loudness changes, or at least that the level difference of these changes when compared to the average noise level is as low as possible. The announcer's voice must be heard, however it must not disturb the surrounding neighborhood. This could be resolved by better distribution and design of the PA system itself. On the other hand, it can be quite challenging to achieve optimal PA system in these open type platforms, which are used in the aforementioned bus station. There are lot of open sound paths and reflections from concrete and asphalt. Trees around the station somewhat reduce the sound level however this is not enough. We have tried exploring and simulating if the signal's dynamics control could help reduce these sudden and unexpected changes [9-11].

For signal analysis we used the Total Distraction Coefficient (TDC) which measures how sudden changes in signal are different to average signal level [8].

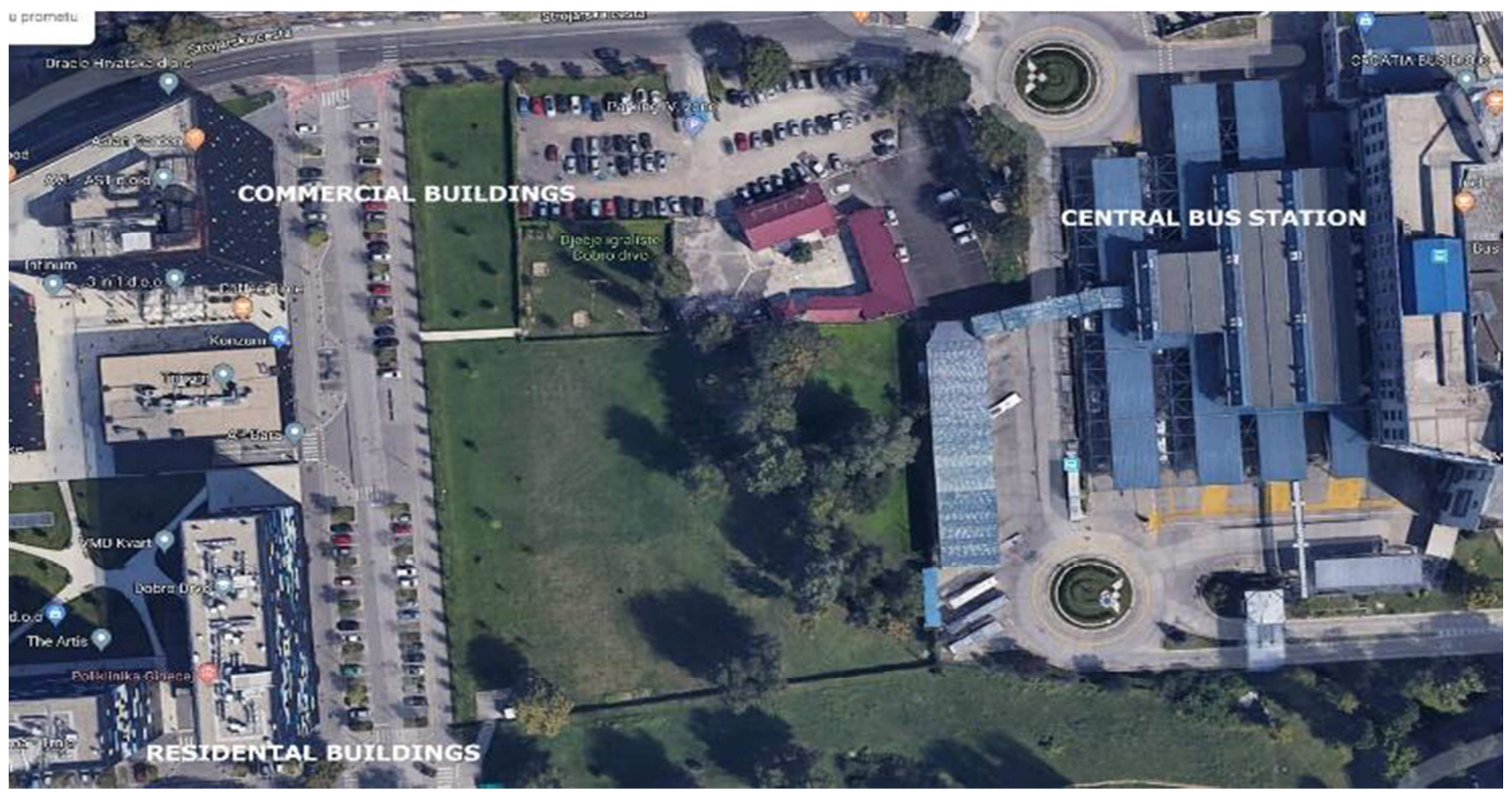

Figure 1. Bird view picture of the neighborhood around the central bus station in Zagreb.

\section{The Proposed Model}

The proposed model predicts a feedback system with microphones installed on surrounding buildings and around the bus station, which will measure emitted noise from the PA system. If the noise exceeds a defined level, microphone system sends a signal in the PA system to reduce loudness. The whole system is based on a compressor-limiter system in order to control the emitted signal level, which will remain above level sufficient for passengers to hear the announcements, however below the level which residents find annoying and disturbing.

The limiter is used to limit the highest sound level and compressor is used to increase subjective loudness and loudness change duration so that the loudness changes could not be perceived as sudden and unexpected. Figure 2 shows a schematic of the proposed model, which includes a PA system with compressor and limiter, loudspeakers on the bus station and microphones on the bus station and surrounding buildings. Signal from the microphones is used as a control signal of the compressor-limiter installed in the PA system. The compressor transfer characteristics should enable the increase of loudness of quiet parts in the announcements in order to be louder than traffic noise, and in addition limit the maximum sound level, especially at the location of buildings. 
LOUDSPEAKERS

ON THE STATION

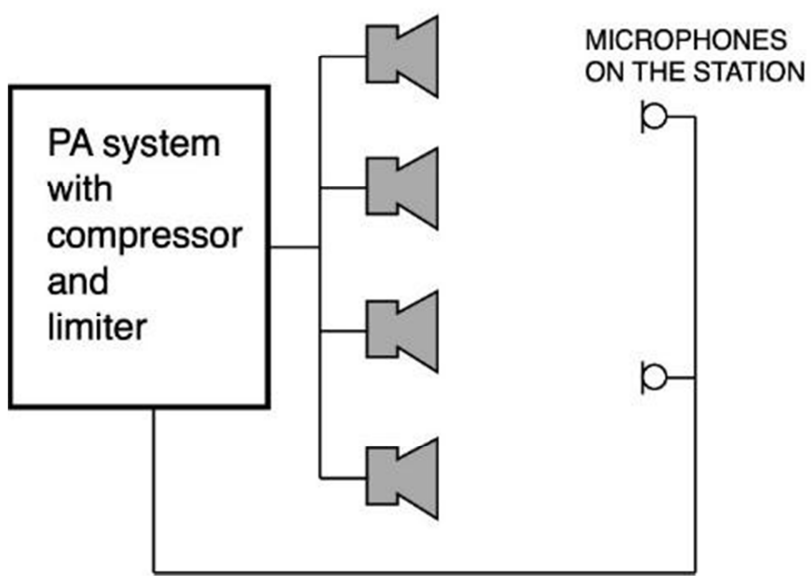

WIRELESS MICROPHONES ON THE BUILDINGS

$\mathrm{p}$

Figure 2. Schematics of a proposed model with a PA system with a compressor-limiter and distributed microphones.

An example of this transfer characteristics is shown in Figure 3. The low-level signal should be amplified more than higher level signals. This will increase the loudness of lowlevel signals at the station however only up to a certain threshold. After this threshold the signal level is kept constant. In order to ensure sudden and unexpected signals are fast compressed, the attack time of the compressor should be short. It is important to note that the system should only work when announcements are made. Otherwise, the system could be triggered with some other sounds which could be picked up by microphones.

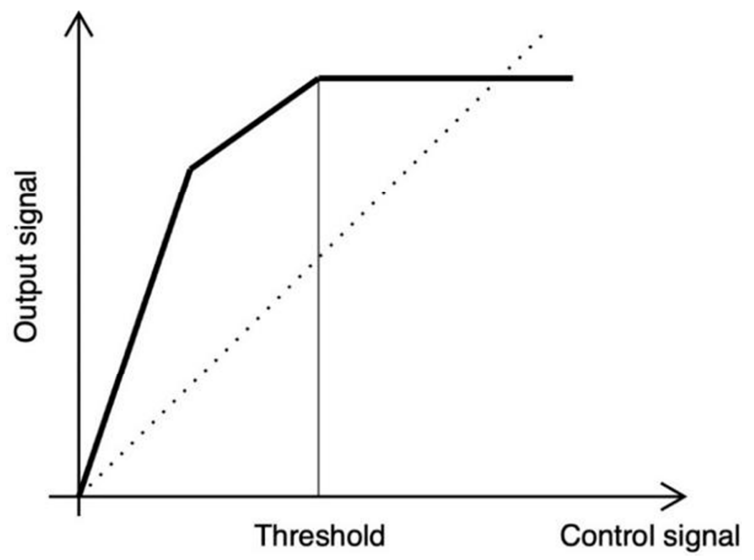

Figure 3. Suggested compressor characteristics.

The lower peak amplitude reduces maximum sound pressure level, which reduces perceived loudness. This can be compensated by higher gain after compression. This would lower the dynamics of the announcement audio signal however it will increase the loudness. This principle is used in today's modern popular music and radio broadcasting [12]. In order to make songs and entire audio programme level louder, audio engineers increase gain of low-level sounds. The research [11] have shown that people perceive highly compressed music as louder. The compressors and limiters in music studios are set for higher gain and relatively high compression. This allows the music to be perceived as louder, which attracts audience. In music, higher compression is used for increasing perceived loudness, which results in lower dynamic range and music reproduction quality [12]. In this case, the higher compression is desired in order to increase the perceived loudness.

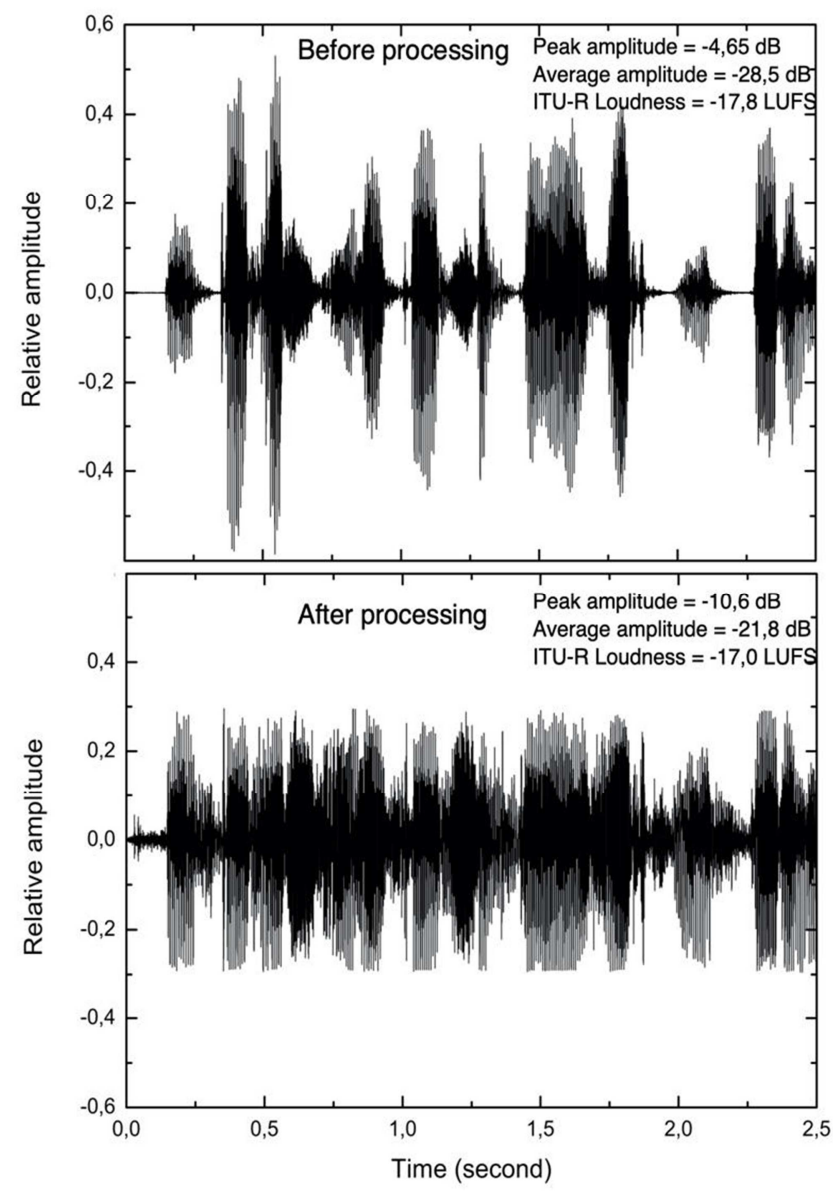

Figure 4. Comparison of an audio signal before and after processing with a compressor with high gain and compression level.

Figure 4 shows an example how perceived loudness can be maintained despite of level decrease. Used audio signal, 
before processing had the peak amplitude of-4.65 dB, average amplitude of- $28.5 \mathrm{~dB}$ and ITU-R loudness od-17.8 LUFS. In 2011, ITU-R organization issued a recommendation for calculation of loudness which takes into account how people perceive loudness [14]. The value, which is given in LUFS units, corresponds to psychoacoustic perception of sound and not on the signal's amplitude. Therefore, signals with lower amplitude could be perceived as louder, depending on their frequency content and dynamics. A can be seen in upper part of the Figure 4, audio signal before processing has larger dynamics. After processing with mentioned compressor characteristics, the signal has lower dynamics and peak amplitude, but perceived loudness remained the same.

Feedforward dynamic signal processor with a Graphical User Interface (GUI) developed in Python is used for the purpose of simulation (shown in Figure 5 [15]). Digital signal processing algorithm is developed in $\mathrm{C}++$ and Cython is used as the interface between the two. The software implementation allows for the transfer function parameters tuning, that is, adjustment of all compressor parameters, like input and output gain, attack and release time, and compression ratio.
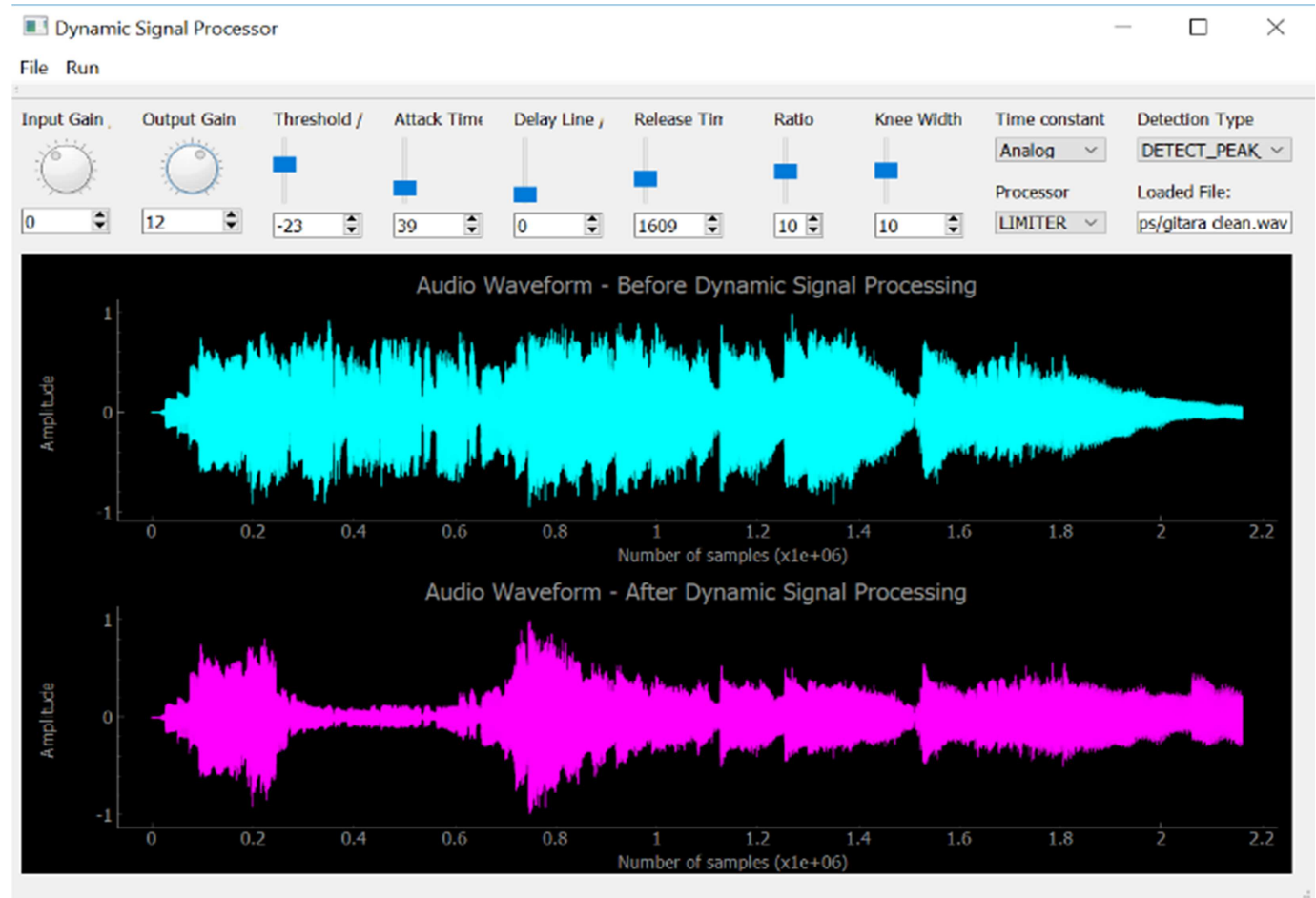

Figure 5. An example of compressor settings with higher output gain and short attack time.

A male voice has been used for reading the announcements of arriving buses mixed together with a background traffic noise. We have compared the level and loudness of overall signal before and after the compressor. Table 1 shows the comparison of the two signals. It can be seen that with the appropriate compressor characteristics the perceived loudness can be maintained with lower signal level, hence lower sound pressure level.

Table 1. Comparison of the announcement audio signal before and after dynamic processing.

\begin{tabular}{lll}
\hline & Before & After \\
\hline Peak amplitude (dB) & -0.95 & -4.9 \\
Average RMS amplitude (dB) & -20.47 & -18.1 \\
ITU-R BS.1770-3 Loudness (LUFS) & -17.9 & -17.1 \\
\hline
\end{tabular}

In this type of PA systems with microphones which could pick up the signal from loudspeakers, it is important to install some kind of an acoustic feedback suppressor [9-11]. This device will reduce a possibility of distortions and high signal levels.

\section{Conclusion}

In this paper we have proposed a model for public announcement systems installed in public places near residential buildings. The proposed model includes a compressor-limiter system controlled by external microphones installed on surrounding buildings. The envisaged idea is the following: when announcements are made through the public announcement system, the signal is processed in order to increase loudness while at the same time decreasing the overall level of emitted sound. Microphones regulate how much announcement signal is above surrounding noise, in order to reduce the residents' annoyance with unexpected and sudden loudness changes.

The study has shown that with the appropriate compressor characteristics and processing of signal's envelope, the sound level could be decreased while retaining the loudness level.

Further work will be focused on additional processing of 
signal, and measurement and analysis of a system installed in an actual public place.

Finally, the results of this research can serve as a basis for future public announcement system in smart cities as they try to improve the overall quality of life in urban areas i.e. they are improving the speech intelligibility while retaining the loudness lever and thus not increasing the noise pollution. Therefore, the residents can benefit from a "cleaner" environment in terms of noise while having their information delivered in a clear and unambiguous way.

\section{References}

[1] W. Passchier-Vermeer and W. F. Passchier, Noise exposure and Public Health, Environmental Health Perspectives, 108, 2000, pp. 123-31.

[2] F. Fahy, Foundations of Engineering Acoustics, Elsevier, 2001

[3] M. Jing, L. Chunjiang, M. Kwan and Y. Chai, A Multilevel Analysis of Perceived Noise Pollution, Geographic Contexts and Mental Health in Beijing, Int J Environ Res Public Health, 15 (7), 2018, pp. 1479.

[4] S. Agarwal and B. L. Swami, Comprehensive approach for the development of traffic noise prediction model for Jaipur city, Environmental Monitoring Assessment, pp. 113-120, 2011.

[5] J. Andersson, A. Oudin, A. Sundström, B. Forsberg, R. Adolfsson and M. Nordin, Road traflc noise, air pollution, and risk of dementia - results from the Betula project, Environmental Research, pp. 334-339, 2018.
[6] M. Arana, R. S. Martin and J. C. Salinas, People exposed to traffic noise in European agglomerations from noise maps, A critical review, Noise Mapping, pp. 40-49, 2014.

[7] https://www.google.com/maps.

[8] M. Suhanek, I. Djurek, S. Grubeša and A. Petošić, Urban Acoustic Environments - An Acoustic Model for Total Distraction Coefficient, Acta Acustica United with Acustica, Volume 105, Number 2, pp. 334-342 (9), 2019.

[9] G. M. Ballou, Handbook for Sound Engineers, 3rd Edition, Focal Press, 2005.

[10] G. Davis and R. Jones, Sound Reinforcement Handbook, Second Edition, Hal-Leonard Corporation, Milwaukee, WI, 1989.

[11] Bob Mc Carthy, Sound Systems: Design and Optimization, Focal Press, New York and London, 2010.

[12] I. Djurek, E. Maganić and M. Suhanek, Music Dynamic Range of FM Radio Stations in Zagreb, Proceedings of the $8^{\text {th }}$ Congress of the Alps Adria Acoustics Association, Zagreb, Croatia, pp. 275-279, 2018.

[13] N. B. H. Croghan, K. H. Arehart and J. M. Kates, Quality and Loudness Judgments for Music Subjected to Compression Limiting, J. Acoust. Soc. Am., Vol. 132, no. 2, pp. 1177-1188, 2012.

[14] ITU-R, "Recommendation ITU-R BS. 1770-3 Algorithms to measure audio programme loudness and true-peak audio level", March 2011.

[15] A. Grgat, Dynamic Audio Signal Processing, MscEE Thesis, February 2019. 